\title{
Constructive Optimization of a Multi-Enzymatic Film based on a Cascade Reaction for Electrochemical Biosensors
}

\author{
Kai Sasaki ${ }^{1,2}$, Hiroyuki Furusawa ${ }^{2,3,4 *}$, Kuniaki Nagamine ${ }^{1,4}$, and Shizuo Tokito ${ }^{1,4 *}$ \\ ${ }^{1}$ Graduate School of Organic Materials Science, Yamagata University, 4-3-16 Jonan, Yonezawa, Yamagata 992-8510 \\ ${ }^{2}$ Innovative Flex Course for Frontier Organic Material Systems (iFront), Yamagata University, 4-3-16 Jonan, Yonezawa, \\ Yamagata 992-8510 \\ ${ }^{3}$ Institute for the Promotion of General Graduate Education (IPGE), Yamagata University, 4-3-16 Jonan, Yonezawa, Yamagata \\ $992-8510$ \\ ${ }^{4}$ Research Center for Organic Electronics (ROEL), Yamagata University, 4-3-16 Jonan, Yonezawa, Yamagata 992-8510 \\ E-mail: hfurusaw@yz.yamagata-u.ac.jp (H.F.); tokito@yz.yamagata-u.ac.jp (S.T.)
}

\section{Supporting Information}

\section{Table of Contents}

Figure S1: Linear plots for the initial rate values of $\Delta E$ measured by (a) the normalsequential layered film electrode, (b) the mixed film electrode, and (c) the reverse-sequential layered film electrode.

Figure S2: Linear plots for the initial rate values of $\Delta E$ measured by (a) the mixed film electrode and (b) the three layered film electrode.

Figure S3: Scanning electron microscopy images of electrode surfaces. 


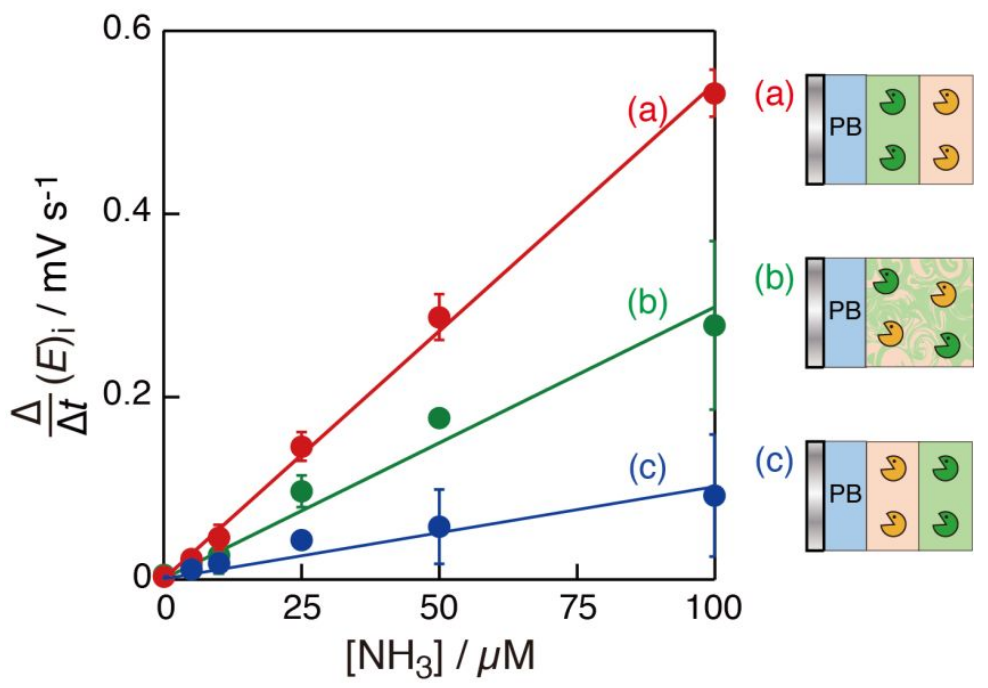

Figure S1. Linear plots for the initial rate values of $\Delta E$ measured by (a) the normal-sequential layered film electrode, (b) the mixed film electrode, and (c) the reverse-sequential layered film electrode. The measurements were carried out at $25^{\circ} \mathrm{C}$ in a $100 \mathrm{mM}$ phosphate buffer solution (pH 8.0) containing $200 \mathrm{mM}$ $\mathrm{KCl}, 2 \mathrm{mM} \mathrm{NADPH}$, and $5 \mathrm{mM} \alpha-\mathrm{KA}$ for the $\mathrm{NH}_{3}$ detection $(5-100 \mu \mathrm{M})$. The error bars indicate the standard deviation $(n=3)$. The lines $\mathrm{a}$ and $\mathrm{b}$ are the same as those in Figure 4B line $\mathrm{d}$ and Figure 3B line $\mathrm{d}$ for ease of comparison, respectively. 


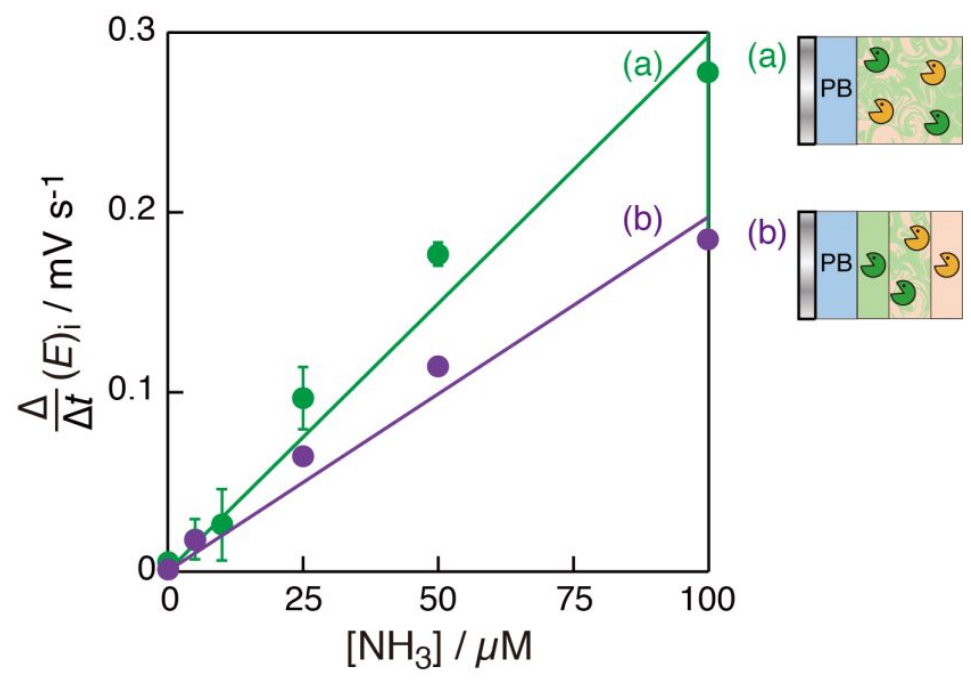

Figure S2. Linear plots for the initial rate values of $\Delta E$ measured by (a) the mixed film electrode and (b) the three layered film electrode. The measurements were carried out at $25^{\circ} \mathrm{C}$ in a $100 \mathrm{mM}$ phosphate buffer solution (pH 8.0) containing $200 \mathrm{mM} \mathrm{KCl}, 2 \mathrm{mM} \mathrm{NADPH}$, and $5 \mathrm{mM} \alpha-\mathrm{KA}$ for the $\mathrm{NH}_{3}$ detection $(5-100$ $\mu \mathrm{M})$. The error bars indicate the standard deviation $(n=3)$. There are no error bars for $(b)$. The line a is the same as that in Figure 3B line d for ease of comparison. 
(A)

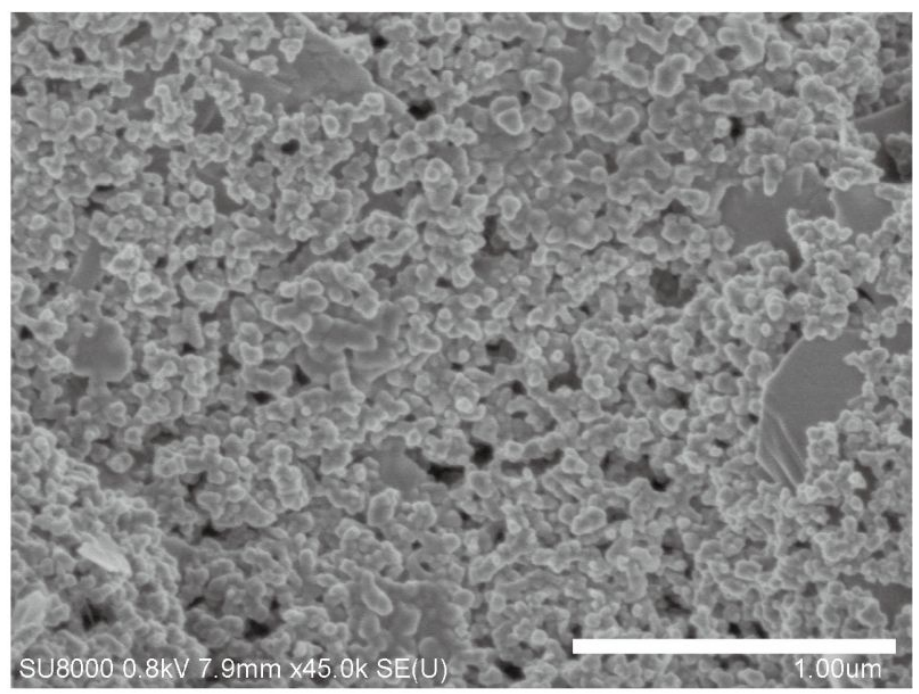

(B)

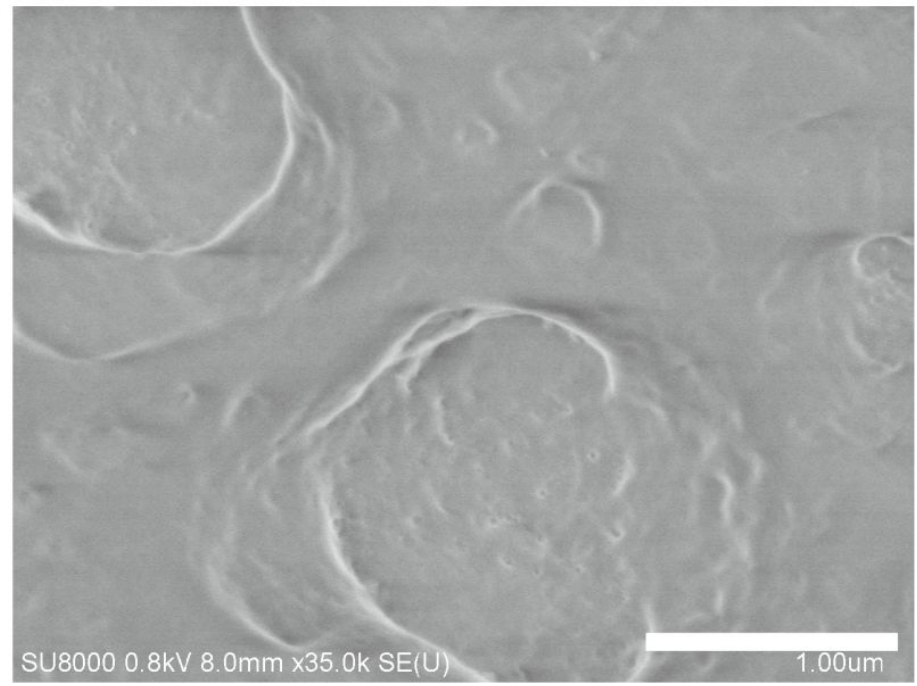

Figure S3. Scanning electron microscopy images of surfaces of (A) a bare PB-carbon electrode and (B) an electrode covered by normal-sequential layered film. The scanning was carried out at acceleration voltage: $0.8 \mathrm{kV}$ using SU8000 (Hitachi High-Tech Co., Ltd., Tokyo, Japan). The white scale bars indicate the $1 \mu \mathrm{m}$ width, respectively. 